\title{
Computed Tomography Radiation Dose: A Primer for Administrators
}

David A. Leswick, Chance S. Dumaine, Nida S. Syed and Derek A. Fladeland

\begin{abstract}
The use of computed tomography (CT) is growing, and, consequently, the associated radiation dose to patients is increasing as well. There is also increasing evidence linking the radiation dose within the range of diagnostic $\mathrm{CT}$ with a significantly increased risk of malignancy. These two factors combine to make radiation dose from diagnostic CT a public health concern.

In order to practise to the best of our abilities and avoid harming patients, the radiation dose from CT must be minimized. Administrators, technologists, radiologists and other physicians are encouraged to work toward this goal through the use of education and a multi-faceted team approach. The objective of this paper is to educate healthcare professionals about the radiation dose from diagnostic $\mathrm{CT}$, including utilization rates, typical examination doses and the risks of this radiation. Our experience in Saskatchewan is discussed. Suggestions regarding CT dose management and optimization are highlighted.
\end{abstract}

T he use of computed tomography (CT) is growing, and, consequently, the associated radiation dose to patients is increasing as well. There is also increasing evidence linking the radiation dose within the range of diagnostic CT with a significantly increased risk of malignancy. These two factors combine to make radiation dose from diagnostic CT a public health concern that reaches beyond the walls of the medical imaging department.

The objective of this paper is to educate healthcare professionals about the radiation from diagnostic CT, including utilization rates, typical examination doses and the risks of this radiation. Our experience in Saskatchewan is discussed. Suggestions regarding CT dose management and optimization are highlighted.

\section{Measures of Radiation Dose}

When reviewing the literature on radiation dose from CT, one thing is abundantly clear: the numerous different measurements and scientific units can be extremely confusing, with many ways to describe the radiation dose from a given procedure. The most commonly used in CT are CT dose index (CTDI), dose length product (DLP) and effective dose. When working at the CT console, two estimates of radiation dose are displayed on most scanners: the CTDI and DLP. CTDI is an estimate of radiation dose along a single slice of the CT, whereas DLP is a better indicator of total patient dose (Coursey and Frush 2008; Huda et al. 2008). Unfortunately, neither CTDI nor DLP are equivalent to dose as they are independent of what is scanned, with no accounting for tissue radiosensitivity (including which organs are scanned and the patient's age) (Aldrich and Williams 2005; 
Coursey and Frush 2008; Huda et al. 2008; McCollough and Schueler 2000).

Effective dose (measured in millisieverts [mSv]) is the sum of the doses to each exposed organ, weighted according to each organ's radiosensitivity (Aldrich et al. 2006; Aldrich and Williams 2005; McCollough and Schueler 2000; Nickoloff et al. 2008). Effective dose allows for the comparison of radiation dose between varying examination types (Aldrich et al. 2006; Aldrich and Williams 2005; Coursey and Frush 2008; Hohl et al. 2006; McCollough and Schueler 2000). It is relatively easily calculated from DLP through use of conversion factors (Coursey and Frush 2008; Sodickson et al. 2009).

Although there are strengths of and benefits to each of these measurements, they can be confusing, particularly for patients, physicians and administrators without a strong background in physics. To help put radiation dose from CT into a better perspective, it can be compared with background environmental radiation (Coursey and Frush 2008; Nickoloff et al. 2008). Background equivalent radiation time (BERT) compares the dose from a procedure to the background radiation to which every member of the public is exposed (Nickoloff et al. 2008). Although background radiation dose is geographically variable, the accepted standard for the purposes of BERT is $3 \mathrm{mSv}$ per year (Nickoloff et al. 2008). Using the concept of BERT, the dose of an average CT abdomen-pelvis scan in Saskatchewan can be expressed as either $15.5 \mathrm{mSv}$ (effective dose) or equivalent to five years and two months of background radiation (BERT) (Leswick et al. 2009) (Table 1).

\section{A major advantage of both}

\section{ultrasonography and MRI is their lack of ionizing radiation. When practical, the use of these alternative modalities should be considered.}

\section{Changes in CT Technology}

The technological revolution of CT has included the development of helical and multi-detector row (MDR) scanning. Slip-ring technology was introduced in 1988 and allows continuous $\mathrm{x}$-ray tube and detector rotation while the patient is moved, to produce helical acquisitions of images (Canadian Institute for Health Information [CIHI] 2007). MDR CT scanners were introduced in 1998, providing another revolutionary jump as multiple images/slices could be acquired simultaneously, resulting in faster imaging times (CIHI 2007). There has since been progressive development of newer MDR scanner designs up to 64 and 256 detector rows (CIHI 2007; Dalrymple et al. 2007; University Health Network [UHN] 2006). It has been found that MDR scanners typically provide higher patient doses than single detector row (SDR) scanners.

An axial CT scan of a patient can be thought of as analogous to cutting a slice of bread from a loaf. Historically, spatial resolution is best within this slice (Dalrymple et al. 2007). With newer scanners, extremely thin slices allow for routine acquisition of isotropic data sets with equivalent resolution in all three imaging

Table 1. Effective dose of chest radiographs, CT scans and atomic bomb survivors with increased risk malignancy*

\begin{tabular}{|l|c|c|c|}
\hline Type of CT Examination & Effective Dose (mSv) & $\begin{array}{c}\text { Equivalent No. of Chest } \\
\text { Radiographs }^{\dagger}\end{array}$ & 1 \\
\hline Chest radiograph $^{\dagger}$ & 0.2 & 14 & $0.8 \mathrm{mo}$ \\
\hline Head $^{\ddagger}$ & 2.7 & 57 & $3 \mathrm{mo}$ \\
\hline Chest $^{\ddagger}$ & 11.3 & 78 & $5 \mathrm{yr}$ and $2 \mathrm{mo}$ \\
\hline Abdomen-pelvis $^{\ddagger}$ & 15.5 & 148 & $9 \mathrm{yr}$ and $10 \mathrm{mo}$ \\
\hline Cumulative CT head, chest and abdomen-pelvis doses $^{\ddagger}$ & 29.5 & 40 & $13 \mathrm{yr}$ and $4 \mathrm{mo}$ \\
\hline Atomic bomb survivors with increased risk malignancy & & & 78 \\
\hline
\end{tabular}

BERT $=$ background equivalent radiation time; $\mathrm{CT}=$ computed tomography.

*Mean effective dose for a two-view chest radiograph; CTs of the head, chest and abdomen-pelvis; combined CT head, chest and abdomen-pelvis; and mean dose of atomic bomb survival with increased risk of malignancy. Chest radiograph equivalent is relative to a $0.2 \mathrm{mSv}$ dose for a general radiological procedure such as a two-view chest radiograph. Please note that the chest radiograph equivalent number may be quoted as greater by a factor of 10 if using $0.02 \mathrm{mSv}$ as the dose from a single view (antero-posterior only) radiograph (University Health Network 2006).

${ }^{\dagger}$ Data from Aldrich and Williams (2005).

${ }^{\ddagger}$ Data from Leswick et al. (2009).

${ }^{\S}$ Data from Brenner and Hall (2007). 
planes (Dalrymple et al. 2007) (Figure 1). These isotropic data sets allow for advanced applications such as CT angiography. Although isotropic data sets can be acquired with little increase in patient dose, if protocols are established with image quality based on the thinnest possible images, patients will be exposed

Figure 1. Computed tomography axial and sagittal images
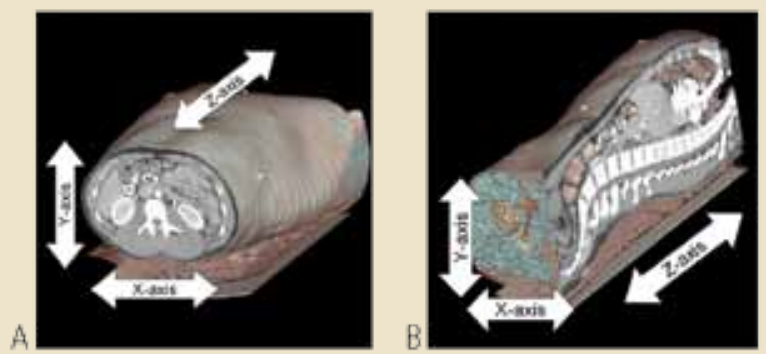

Computed tomography (CT) study to reassess the aorta of an 18-year-old male involved in a motor vehicle accident. Presented images are three-dimensional (3-D) reformatted images built from $1.25 \mathrm{~mm}$ axial source images during the arterial phase of intravenous contrast. $A$, The "traditional" axial image at the level of the kidneys and liver. $B, A$ reconstructed sagittal image along the plane of the spine possible with this near-isotropic data set. Both images are presented along with a companion 3-D reformat. No injuries are visible on these presented images. The effective dose for this examination was $17.3 \mathrm{mSv}$. The patient also underwent a routine CT of the chest, abdomen and pelvis four hours earlier, with an effective dose of 14.6 $\mathrm{mSv}$, resulting in an total one-day CT dose of $31.9 \mathrm{mSv}$ (10 years and seven months BERT). The scan was performed with a GE Lightspeed Ultra 8 detector row scanner with advanced processing, performed on a GE Advantage Workstation, Version 4.4.

to a significantly higher radiation dose (Dalrymple et al. 2007). In addition to isotropic data sets, newer CT units allow for faster scanning times. This has expanded the use of CT in children as faster scanning times reduce the need for anesthesia or sedation (Brenner and Hall 2007). Although the decreased need for anesthesia is a patient safety benefit, it should be remembered that the radiation risks are highest in children (Brenner and Hall 2007; Coursey and Frush 2008).

\section{CT Use in Canada}

Around the world, the use of CT is steadily increasing, with Canada being no exception. In 1990, there were 198 CT scanners in Canada, which more than doubled to 419 scanners by 2007 (CIHI 2007). Canadians are also being scanned more frequently, with a $28 \%$ increase in the number of CT examinations per 1,000 population in the three years leading up to the 2006-2007 fiscal year (Table 2) (CIHI 2007). To put this use in a global context, Canada performs 103.3 CT scans per 1,000 population annually versus 207.4 scans in the United States and 53.7 scans in England (CIHI 2007).

Canadian CT scanner technology is also improving. Approximately 90\% of Canadian scanners were MDR scanners in 2007 (CIHI 2007). Of these MDR scanners, 60\% had greater than 16 detector rows and $28 \%$ had at least 64 detector rows (CIHI 2007). This can be contrasted with the 19 scanners in a 2004 British Columbia dose survey, all of which had 16 detector rows or less (Aldrich et al. 2006).

\section{CT and Cancer Risk}

The exact relationship between low-level radiation doses, such as those from CT scans, and cancer is uncertain and may never be known. However, the most likely effect is radiation-induced carcinogenesis (Brenner and Hall 2007). An analysis of the evidence to date led the Biological Effects of Ionizing Radiation (BEIR) VII report, Health Risks from Exposure to Low Levels of Ionizing Radiation, to recommend no threshold as the most reasonable description of the relationship between low-dose exposure and cancer incidence (National Research Council 2006). This means that even low-level radiation doses, such as those from CT, can induce malignancy.

Much of the evidence to date linking radiation dose and malignancy in humans comes from a study on 25,000 survivors of the atomic bombs dropped on Japan in 1945 (Brenner and Hall 2007). There has been a significant increase in the overall risk of cancer for those survivors, who received an average radiation dose of $40 \mathrm{mSv}(5-150 \mathrm{mSv}$ range) (see Table 1) (Brenner and Hall 2007). A study of more than 400,000 radiation industry workers exposed to an average dose of $20 \mathrm{mSv}$ also showed an association between dose and cancer mortality (Brenner and Hall 2007).

These doses are near the range of diagnostic CT, particularly when considering that patients may require scans of more than one body part and often have repeated imaging (see Tables 1 and 2). Based on average dose levels, a patient undergoing CT scans of the head, chest and abdomen-pelvis at the same visit may experience $29.5 \mathrm{mSv}$ dose (see Table 1) (Leswick et al. 2009). A clinical example of multi-body part repeat scanning is the 18 -year-old patient in Figure 1, with a single-day dose of $31.9 \mathrm{mSv}$.

Another important concern is that patients often receive multiple CT examinations over their lifetime. Sodickson et al. (2009) examined the imaging histories of over 30,000 predominantly adult patients scanned at their institution in 2007. Over greater than 20 years, $33 \%$ of patients received greater than five scans, $5 \%$ received greater than 22 scans and $1 \%$ received greater than 38 scans (Sodickson et al. 2009) (see Table 2). This corresponded to $15 \%$ of patients exposed to greater than $100 \mathrm{mSv}$ and $4 \%$ of patients exposed to more than $250 \mathrm{mSv}$ (Sodickson et al. 2009) (see Table 2).

Although the risks for most patients are small, the increasing 
Table 2. By the numbers: Interesting percentages

\begin{tabular}{|c|c|}
\hline Percentage & Statistic \\
\hline $0.7-2$ & Percent of cancers in the United States attributable to CT (Brenner and Hall 2007; Sodickson et al. 2009) \\
\hline 53 and 61 & $\begin{array}{l}\text { Percent of emergency physicians and radiologists who, in a } 2004 \text { survey, thought the average CT scan had a dose } \\
\text { less than that of } 10 \text { chest radiographs (Lee et al. 2004). Actual answer is } 50-71 \text { two-view chest radiographs. } \\
\text { (Leswick et al. 2009) }\end{array}$ \\
\hline 91 and 53 & $\begin{array}{l}\text { Percent of emergency physicians and radiologists who thought there was no risk of malignancy from CT in a } 2004 \\
\text { survey (Lee et al. 2004) }\end{array}$ \\
\hline 28 & $\begin{array}{l}\text { Percent increase in number of CT examinations per 1,000 Canadian population from 2003-2004 to 2006-2007 (CIHI } \\
\text { 2007) }\end{array}$ \\
\hline 33 and 5 & $\begin{array}{l}\text { Percent of patients with more than five and } 22 \text { CT scans, respectively, in }>20 \text { years of their imaging history } \\
\text { (Sodickson et al. 2009) }\end{array}$ \\
\hline 15 and 4 & $\begin{array}{l}\text { Percent of patients with }>100 \mathrm{mSv} \text { and } 250 \mathrm{mSv} \text { radiation dose, respectively, over }>20 \text { years of their imaging history } \\
\text { (Sodickson et al. 2009) }\end{array}$ \\
\hline 6 & $\begin{array}{l}\text { Percent of cancers estimated to be attributed to CT among the } 1 \% \text { of patients with highest risk over }>20 \text { years of } \\
\text { their imaging history (Sodickson et al. 2009) }\end{array}$ \\
\hline $14-32 \%$ & Percent dose reduction observed through use of ATCM in chest and abdomen-pelvis CT (Mulkens et al. 2005) \\
\hline 17 and 31 & $\begin{array}{l}\text { Provincial-wide percent decrease in dose from single-phase abdomen-pelvis and chest on MDR scanners via } \\
\text { protocol optimization at the various sites in Saskatchewan (Dumaine et al. 2009) }\end{array}$ \\
\hline
\end{tabular}

ATCM = automatic tube current modulation; $\mathrm{CIHI}=$ Canadian Institute for Health Information; $\mathrm{CT}=$ computed tomography; $\mathrm{MDR}=$ multi-detector row .

population dose may be a public health issue in the future (Brenner and Hall 2007). Based on 1991-1996 data, it was estimated that $0.4 \%$ of all cancers in the United States may be attributable to radiation from CT studies (Brenner and Hall 2007). Adjusting for current use, it can now be estimated that between 0.7 and $2 \%$ of cancers in the United States are secondary to CT radiation (see Table 2) (Brenner and Hall 2007; Sodickson et al. 2009).

Unfortunately, knowledge regarding CT's radiation risk has lagged behind the patterns of increasing dose. In a 2004 survey; the majority of emergency physicians and radiologists vastly underestimated the dose of a CT scan and did not believe there was risk of malignancy from CT (see Table 2) (Lee et al. 2004). It can only be hoped that in the five years since this study was published, referring physician and radiologist knowledge has improved.

\section{CT in Saskatchewan}

Saskatchewan's population was just over one million in 2008 (Saskatchewan Bureau of Statistics 2008). There are currently 15 CT scanners in Saskatchewan, two of which are used exclusively for cancer therapy (CIHI 2007). Eight of Saskatchewan's scanners have been purchased within the past four years, four of which were new installations in communities previously without CT. However, scanner technology in Saskatchewan remains rather limited, with only $40 \%$ of the CTs having technology higher than 10 detector rows (the third-lowest percentage in Canada) (CIHI 2007). The annual number of patient CT examinations in Saskatchewan is also rising; in the three years leading up to the 2006-2007 fiscal year, the number of annual CT scans increased by $58 \%$ in Saskatchewan, versus a $32 \%$ increase for Canada (CIHI 2007).

In 2006, we investigated the current patient doses across Saskatchewan. Twelve of the province's 13 diagnostic CT scan centres participated in a survey of patient doses (Leswick et al. 2009). CT technologists across the province recorded measurements of dose on just over 2,000 scans (Leswick et al. 2009). Effective doses for head, chest and combined abdomen-pelvis scans were $2.7 \mathrm{mSv}, 11.3 \mathrm{mSv}$ and $15.5 \mathrm{mSv}$, respectively (see Table 1) (Leswick et al. 2009). These doses are similar to those in a 2004 British Columbia survey on doses (Aldrich et al. 2006; Leswick et al. 2009). Doses from MDR scanners were more than double the doses from single-detector row scanners, illustrating the dose difference between these technologies (Leswick et al. 2009). 
A follow-up dose survey was performed in Saskatchewan in 2008 (Dumaine et al. 2009). Although overall mean effective doses for chest and abdomen-pelvis scans were similar, there was a $31 \%$ and $17 \%$ decrease for MDR scanner single-phase chest and abdomen-pelvis examinations, respectively (see Table 2) (Dumaine et al. 2009). This was done entirely through protocol optimization at the participating sites as there were no new installations between the two surveys. CT head examinations showed a slight dose increase, mainly because of radiologist preference at a few institutions (Dumaine et al. 2009).

\section{Dose Management}

There are several methods by which radiologists and technologists can reduce the dose from a CT scan. These include modifying the tube current (milliampere $[\mathrm{mA}]$ ), peak voltage (kilovolts peak $[\mathrm{kVp}]$ ), table pitch and gantry rotation time, collimation and limiting the number of phases acquired. Excellent reviews of these techniques are described elsewhere (Brenner and Hall 2007; Coursey et al. 2008; Frush and Applegate 2004; Kalra et al. 2004b). Three methods of dose reduction need to be specifically addressed: automatic tube current modulation (ATCM), patient shielding and decreasing number of studies by eliminating unnecessary examinations and using alternative tests.

\section{Automatic Tube Current Modulation}

ATCM is a dose-modulation technique available on most modern CT scanners, although its vendor-specific name varies between companies and sometimes between models. ATCM systems work by the general principle of adjusting the tube current according to the size, density and geometry of the part of the body being scanned (Althen 2005; Kalra et al. 2004a; McNitt-Gray 2002).

Studies of the effectiveness of ATCM systems are difficult as performance varies based on radiologist and technologist choices (Leswick et al. 2008; Mulkens et al. 2005). With that in mind, previous studies have shown dose reduction in the range of 14-32\% for chest and abdomen-pelvis scans (see Table 2) (Mulkens et al. 2005). These dose reductions emphasize the potential value of ATCM systems, although they must be used with caution when obtaining isotropic data sets (Dalrymple et al. 2007).

\section{Patient Shielding}

There are two major types of shielding used in CT: in-plane shielding and shielding of non-imaged tissues outside the scan field of view. In-plane shielding uses material placed over radiosensitive tissues within the imaged area of the body providing local dose reduction while still allowing for diagnostic images. These in-plane bismuth shields can provide dose reductions ranging from approximately 25 to $75 \%$ for eyes, thyroid and breast tissue (Coursey and Frush 2008; Coursey et al. 2008;
Hohl et al. 2006; Hopper 2002; Hopper et al. 1997; Leswick et al. 2008). When combined with ATCM, shields should be placed after the scout scan is acquired (Coursey et al. 2008; Leswick et al. 2008).

Even though the $\mathrm{x}$-ray beam in CT is well collimated to the region being scanned, there is some exposure to adjacent body parts (Neeman et al. 2006). For this reason, shielding body parts adjacent to the scanned field of view may be advisable. An example is shielding the abdomen and pelvis of a patient receiving a chest CT, something particularly attractive for pregnant patients. By using these shields, dose reductions can be accomplished in the range of $40-92 \%$ for these adjacent body parts (Kennedy et al. 2007; Neeman et al. 2006).

\section{Reducing Number of CT Scans and Using Alternative Modalities}

CT, ultrasonography and magnetic resonance imaging (MRI) are complementary modalities with overlap in their imaging abilities. A major advantage of both ultrasonography and MRI is their lack of ionizing radiation. When practical, the use of these alternative modalities should be considered. This can be highlighted by reviewing the clinical scenario of suspected appendicitis.

Appendicitis is the most common acute abdominal disorder suspected in emergency departments (Gaitini et al. 2008). Because of its high sensitivity, specificity and the ability to visualize a normal appendix (thus ruling out the diagnosis of appendicitis), CT has become the primary screening modality for appendicitis at many institutions. Although ultrasonography has a lower sensitivity for appendicitis than CT $(74 \%$ versus $100 \%$ ), it does have a high specificity $(97 \%)$ and negative predictive value $(93 \%)$, which make it a good screening test prior to CT (Gaitini et al. 2008). An "ultrasonography first" imaging protocol for appendicitis reserves CT for cases with inconclusive ultrasonographic findings or according to clinical need following ultrasonography. A review of an institution with an ultrasonography first policy found that nearly $70 \%$ of suspected appendicitis patients could be managed with ultrasonography alone (Gaitini et al. 2008).

Appendicitis is a particularly difficult clinical diagnosis in pregnant patients, a population where radiation dose to the fetus is a major concern. Additionally, ultrasonography may be difficult because of shifting of the appendix by the gravid uterus. MRI can examine the appendix without radiation, with early studies showing the favourable performance of MRI in pregnant patients with suspected appendicitis (Israel et al. 2008).

\section{Recommendations}

Despite the radiation risks, CT remains an invaluable diagnostic tool for modern medicine. However, to justify a CT scan, the potential or proven benefits should outweigh the risks to the 
patient (Sodickson et al. 2009; UHN 2006). The following recommendations can be made with a goal of providing appropriate use of $\mathrm{CT}$ while minimizing risks of the scans we perform.

\section{Make Radiation Dose Management a Priority for All CT Scanners}

CT manufacturers now have numerous approaches to help manage dose (Althen 2005). Radiation dose management should be a primary concern when purchasing new equipment as it is equivalent to patient safety.

However, radiation dose management is not only a concern for new CT purchases. Protocol choice can cause significant variations in dose provided by similar or even identical scanners (Koller et al. 2003; UHN 2006). The Saskatchewan decreases in single-phase scan chest and abdomen-pelvis CT dose on the same MDR scanners between 2006 and 2008 highlight the importance of user-selected scan parameters in CT dose management (Dumaine et al. 2009). With this in mind, technologists, radiologists and administrators should make optimization of protocols toward lower dose a priority for all active CT scanners.

\section{Archive Patient Dose Data}

The CTDI and DLP are displayed on modern CT scanners when planning cases and as a summary after the scan is completed. Unfortunately, some institutions do not routinely archive these data. CT dose can be thought of as analogous to a dose of a medication (Coursey and Frush 2008) and, as such, should be archived as part of the patient's medical record. Archiving these data will also help identify patients requiring repeated imaging who have a greater risk of cancer induction and might benefit the most from dose-reduction strategies or the use of alternative imaging modalities (Sodickson et al. 2009). Furthermore, archiving these data will make audits of dose possible.

\section{Perform Regular Audits of CT Dose and Compare with Reference Standards}

Unless dose audits are performed, institutions (including radiologists, technologists and administrators) have no way of knowing what level of dose their patients are being exposed to. The establishment of reference levels, such as those used in the United Kingdom, has been found to be an effective way of reducing patient dose in CT (UHN 2006). Although variation in dose is expected as examinations must be tailored to the clinical indication and patient anatomy, consistent dose levels above reference levels can identify problems with either equipment or protocols (Nickoloff et al. 2008).

\section{Share Low-Dose Protocols between Institutions}

Lowering dose should be a priority for every institution. Unfortunately, optimizing protocols requires familiarity with the specific technology at an institution (Coursey and Frush
2008). The level of familiarity needed may be greater than that achievable by many radiologists and technologists because of a busy clinical workload and other demands on a practitioner's continuing medical education time. This is evidenced by the number of radiologists who both underestimated CT dose and its potential effects in a 2004 survey (Lee et al. 2004).

The Ontario report on CT safety issues (UHN 2006) recommended the development of methods to share best practice CT protocols between health institutions. By sharing such protocols, all institutions can use low-dose protocols. This should include the use of ATCM systems and shielding when appropriate (Brenner and Hall 2007; Coursey and Frush 2008; UHN 2006).

\section{Radiation dose from CT is increasing, with doses within the range of that seen in atomic bomb survivors.}

\section{Empower Radiologists to Act as Consultants}

One of the best ways to decrease radiation dose to the population is to avoid unnecessary tests or to use alternative imaging modalities (Brenner and Hall 2007; Sodickson et al. 2009). Health regions need to empower and support radiologists working in consultation with ordering physicians to decrease unnecessary examinations and use alternative examinations with no ionizing radiation.

\section{Ensure Sufficient Access to Complementary Imaging Modalities}

In order to effectively use alternative imaging modalities, there must be appropriate access. MRI and ultrasonography have overlapping clinical indications with CT (Brenner and Hall 2007; CIHI 2007). To be viable alternatives to CT, health regions should have adequate resources to support the realistic use of MRI and ultrasonography in a time-effective manner for appropriate patient evaluation. If ultrasonography or MRI is not available at an institution, relationships should be built with other institutions that can offer these resources.

\section{Educate Non-radiologist Physicians}

In our Canadian system, CT scans are ordered by physicians other than radiologists. Even if radiologists, technologists and administrators work to minimize dose from CT protocols, the best way to decrease exposure is by not performing the scan at all if alternative methods of examination are available (Brenner and Hall 2007). Given that physicians should weigh the risks and benefits of any test they order and referring physicians underestimate the risk of CT radiation dose (Lee et al. 2004; UHN 2006), there is a need for education about the risks of 
CT. Administrators should work with radiologists and other physicians to facilitate this education.

\section{Employ Teamwork}

A single radiologist, technologist, referring physician or administrator cannot change radiation dose alone. By working as a team both within an institution and between sites, healthcare professionals can ensure that $\mathrm{CT}$ dose is minimized for the population as a whole.

\section{Conclusion}

Radiation dose from CT is increasing, with doses within the range of that seen in atomic bomb survivors (Brenner and Hall 2007; Leswick et al. 2009). This is a public safety concern as up to $2 \%$ of cancers in North America may be caused by CT (Brenner and Hall 2007; UHN 2006).

The prototypical healthcare oath is the Hippocratic oath, a document dating back 24 centuries. One of the central tenants of this oath is "to practice and prescribe to the best of my abilities for the good of my patients, and to try and avoid harming them." In order to practise to the best of our abili- ties and avoid harming patients, the radiation dose from CT must be minimized. Administrators, technologists, radiologists and other physicians are encouraged to work toward this goal through the use of education and a multi-faceted team approach. HQ

\section{References}

Aldrich, J.E., A.M. Bilawich and J.R. Mayo. 2006. "Radiation Doses to Patients Receiving Computed Tomography Examinations in British Columbia." Canadian Association of Radiologists Journal 57(2): 79-85.

Aldrich, J.E. and J. Williams. 2005. "Change in Patient Doses from Radiological Examinations at the Vancouver General Hospital, 1991-2002." Canadian Association of Radiologists Journal 56(2): 94-99.

Althen, J.N. 2005. "Automatic Tube-Current Modulation in CT A Comparison between Different Solutions." Radiation Protection Dosimetry 114(1-3): 308-12.

Brenner, D.J. and E.J. Hall. 2007. "Computed Tomography - An Increasing Source of Radiation Exposure." New England Journal of Medicine 357(22): 2277-84.

Canadian Institute for Health Information. 2007. Medical Imaging in Canada 2007. Ottawa, ON: Author.

Coursey, C.A. and D.P. Frush. 2008. "CT and Radiation: What

\section{We value our contribution to the health of Canadians and to the health system through the leadership excellence of our members.}

\section{Nous valorisons notre apport à la santé des canadiens et au système de soins de santé par lexcellence du leadership de nos membres.}

\section{Access to Professional Networks \\ Healthcare Industry Updates \\ Professional Development \\ Industry Recognition}

\section{Accès à des réseaux professionnels}

Mises à jour dans le domaine de la santé Perfectionnement professionnel Reconnaissance dans le domaine www.cchse.org

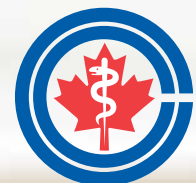

Canadian College of Health Service Executives Collège canadien des directeurs de services de santé

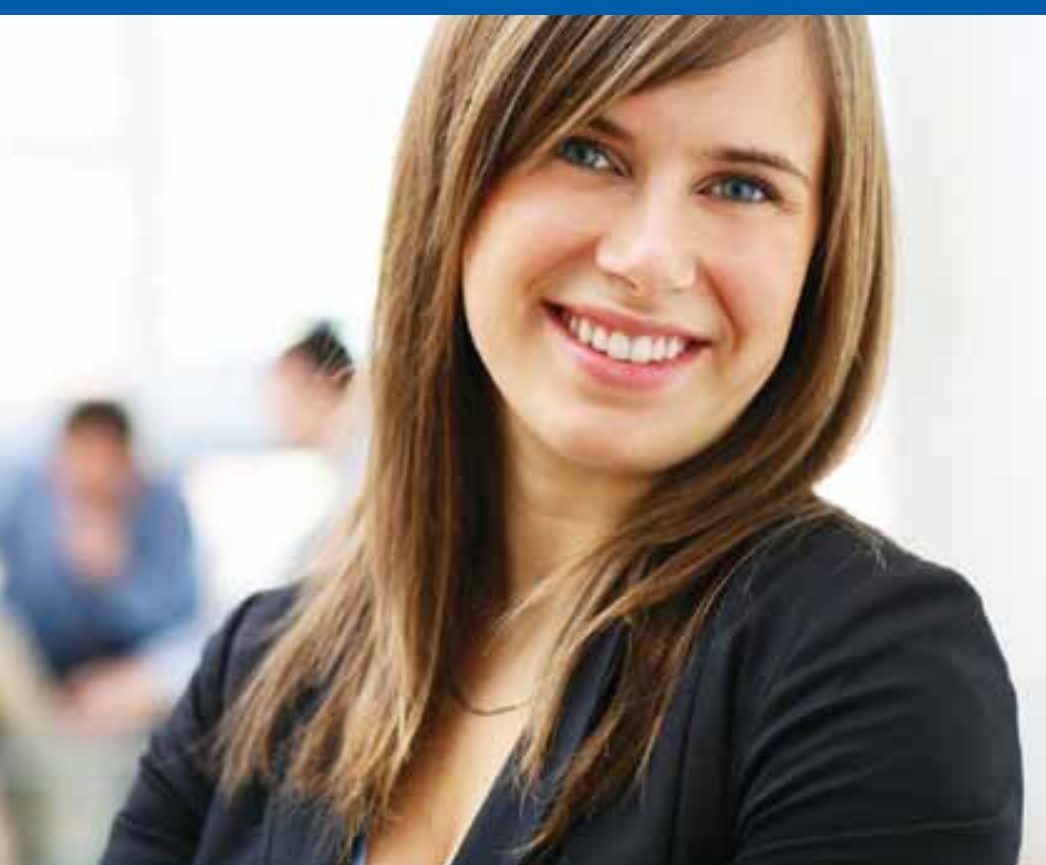


Radiologists Should Know.” Applied Radiology 13(3): 22-29.

Coursey, C., D.P. Frush, T. Yoshizumi, G. Toncheva, G. Nguyen and S.B. Greenberg. 2008. "Pediatric Chest MDCT Using Tube Current Modulation: Effect on Radiation Dose with Breast Shielding." AJR American Journal of Roentgenology 190(1): W54-61.

Dalrymple, N.C., S.R. Prasad, F.M. El-Merhi and K.N. Chintapalli. 2007. "Price of Isotropy in Multidetector CT." Radiographics 27(1): 49-62.

Dumaine, C.S., D.A. Leswick, D.A. Fladeland, H.J. Lim and L.J. Toews. 2009. Improving Radiation Dose from Diagnostic CT Examinations in Saskatchewan. Poster presented at the Canadian Association of Radiologists 72nd Annual Scientific Meeting. Montreal, Quebec.

Frush, D.P. and K. Applegate. 2004. "Computed Tomography and Radiation: Understanding the Issues." Journal of the American College of Radiology 1(2): 113-19.

Gaitini, D., N. Beck-Razi, D. Mor-Yosef, D. Fischer, O. Ben Itzhak, M.M. Krausz and A. Engel. 2008. "Diagnosing Acute Appendicitis in Adults: Accuracy of Color Doppler Sonography and MDCT Compared with Surgery and Clinical Follow-Up." AJR American Journal of Roentgenology 190(5): 1300-06.

Hohl, C., J.E. Wildberger, C. Suss, C. Thomas, G. Muhlenbruch, T. Schmidt, D. Honnef, R.W. Günther and A.H. Mahnken. 2006. "Radiation Dose Reduction to Breast and Thyroid during MDCT: Effectiveness of an In-Plane Bismuth Shield." Acta Radiologica 47(6): 562-67.

Hopper, K.D. 2002. "Orbital, Thyroid, and Breast Superficial Radiation Shielding for Patients Undergoing Diagnostic CT." Seminars in Ultrasound, CT, and MR 23(5): 423-27.

Hopper, K.D., S.H. King, M.E. Lobell, T.R. TenHave and J.S. Weaver. 1997. "The Breast: In-Plane X-Ray Protection during Diagnostic Thoracic CT - Shielding with Bismuth Radioprotective Garments." Radiology 205(3): 853-58.

Huda, W., K.M. Ogden and M.R. Khorasani. 2008. "Converting Dose-Length Product to Effective Dose at CT." Radiology 248(3): 995-1003.

Israel, G.M., N. Malguria, S. McCarthy, J. Copel and J. Weinreb. 2008. "MRI vs. Ultrasound for Suspected Appendicitis during Pregnancy." Journal of Magnetic Resonance Imaging 28(2): 428-33.

Kalra, M.K., M.M. Maher, T.L. Toth, B. Schmidt, B.L. Westerman, H.T. Morgan and S. Saini. 2004a. "Techniques and Applications of Automatic Tube Current Modulation for CT.” Radiology 233(3): 649-57.

Kalra, M.K., M.M. Maher, T.L. Toth, L.M. Hamberg, M.A. Blake, J.A. Shepard and S. Saini. 2004b. "Strategies for CT Radiation Dose Optimization." Radiology 230(3): 619-28.

Kennedy, E.V., G.R. Iball and D.S. Brettle. 2007. "Investigation into the Effects of Lead Shielding for Fetal Dose Reduction in CT Pulmonary Angiography." British Journal of Radiology 80(956): 631-38.

Koller, C.J., J.P. Eatough and A. Bettridge. 2003. "Variations in Radiation Dose between the Same Model of Multislice CT Scanner at Different Hospitals." British Journal of Radiology 76(911): 798-802.

Lee, C.I., A.H. Haims, E.P. Monico, J.A. Brink and H.P. Forman. 2004. "Diagnostic CT Scans: Assessment of Patient, Physician, and Radiologist Awareness of Radiation Dose and Possible Risks." Radiology 231(2): 393-98.

Leswick, D.A., M.M. Hunt, S.T. Webster and D.A. Fladeland. 2008.
"Thyroid Shields versus Z-Axis Automatic Tube Current Modulation for Dose Reduction at Neck CT.” Radiology 249(2): 572-80.

Leswick, D.A., N.S. Syed, C.S. Dumaine, H.J. Lim and D.A. Fladeland. 2009. "Radiation Dose from Diagnostic Computed Tomography in Saskatchewan." Canadian Association of Radiologists Journal 60(2): 71-78.

McCollough, C.H. and B.A. Schueler. 2000. "Calculation of Effective Dose." Medical Physics 27(5): 828-37.

McNitt-Gray, M.F. 2002. "AAPM/RSNA Physics Tutorial for Residents: Topics in CT. Radiation Dose in CT." Radiographics 22(6): 1541-53.

Mulkens, T.H., P. Bellinck, M. Baeyaert, D. Ghysen, X. Van Dijck, E. Mussen, C. Venstermans and J.L. Termote. 2005. "Use of an Automatic Exposure Control Mechanism for Dose Optimization in Multi-detector Row CT Examinations: Clinical Evaluation.” Radiology 237(1): 213-23.

National Research Council (US), Committee to Assess Health Risks from Exposure to Low Level of Ionizing Radiation. 2006. Health Risks from Exposure to Low Levels of Ionizing Radiation : BEIR VII Phase 2. Washington, DC: National Academies Press.

Neeman, Z., S.A. Dromi, S. Sarin and B.J. Wood. 2006. "CT Fluoroscopy Shielding: Decreases in Scattered Radiation for the Patient and Operator." Journal of Vascular and Interventional Radiology 17(12): 1999-2004.

Nickoloff, E.L., Z.F. Lu, A.K. Dutta and J.C. So. 2008. "Radiation Dose Descriptors: BERT, COD, DAP, and Other Strange Creatures." Radiographics 28(5): 1439-50.

Saskatchewan Bureau of Statistics. 2008. Population. Regina, SK: Author. Retrieved December 19, 2008. <http://www.stats.gov.sk.ca/ pop/popindex.php>.

Sodickson, A., P.F. Baeyens, K.P. Andriole, L.M. Prevedello, R.D. Nawfel, R. Hanson and R. Khorasani. 2009. "Recurrent CT, Cumulative Radiation Exposure, and Associated Radiation-Induced Cancer Risks from CT of Adults.” Radiology 251(1): 175-84.

University Health Network, Centre for Global eHealth Innovation, Healthcare Human Factors Group. 2006. Computed Tomography Radiation Safety Issues in Ontario. Toronto, ON: Author.

\section{About the Authors}

David A. Leswick, MD, FRCPC, is a member of the Department of Radiology, Royal University Hospital, University of

Saskatchewan, Saskatoon, Saskatchewan. You can reach him by phone at 306-655-2371, by fax at 306-655-2370 or by e-mail at david.leswick@saskatoonhealthregion.ca.

Chance S. Dumaine is a medical student at the College of Medicine, University of Saskatchewan.

Nida S. Syed, MD, is a member of the Department of Radiology at McMaster University, in Hamilton, Ontario.

Derek A. Fladeland, MD, FRCPC, is a member of the Department of Radiology, Royal University Hospital, University of Saskatchewan. 


\section{The best ideas are common property.*}

The editors and authors acknowledge the support of the Canadian Patient Safety Institute, the Health Council of Canada and Accreditation Canada. They are committed to sharing these ideas, policies and practices to help make patient healthcare safer. Their support motivated the authors, encouraged the editors and now benefit the researchers, the policy makers, the administrators and the care providers who have free and unlimited access to this important literature. It is now common property. Thank you.

The preparation and presentation of this issue of Patient Safety Papers was enabled by these supporting organizations:
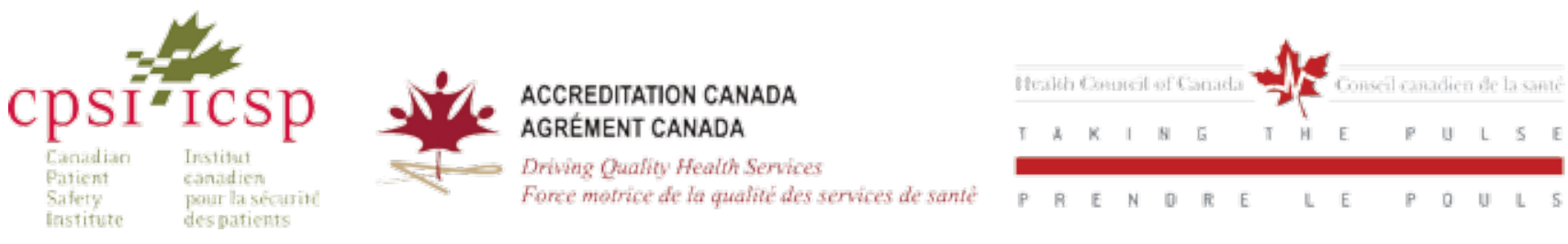

\section{Baxter $1 \mathrm{BD}$}
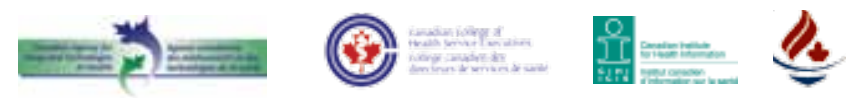

CANADLAN

NURSES

ASSOCINTION

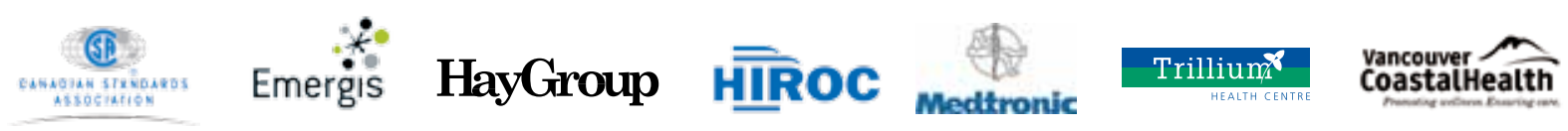

